\title{
ARTICLE \\ One-day tropisetron treatment improves cognitive deficits and P50 inhibition deficits in schizophrenia
}

\author{
Luyao Xia ${ }^{1,2}$, Lei Liu ${ }^{3}$, Xiaohong Hong ${ }^{3}$, Dongmei Wang ${ }^{1,2}$, Gaoxia Wei ${ }^{1,2}$, Jiesi Wang ${ }^{1,2}$, Huixia Zhou ${ }^{1,2}$, Hang Xu ${ }^{1,2}$, Yang Tian ${ }^{1,2}$, \\ Qilong Dai ${ }^{1,2}$, Hanjing E. Wu ${ }^{4}$, Catherine Chang ${ }^{4}$, Li Wang ${ }^{1,2}$, Thomas R. Kosten ${ }^{5}$ and Xiang Yang Zhang $\mathbb{B}^{1,2}$
}

The core features of schizophrenia (SCZ) include cognitive deficits and impaired sensory gating represented by P50 inhibition deficits, which appear to be related to the a7 nicotinic acetylcholine receptor (nAChR). An agonist of nAChR receptor may improve these defects. This study aimed to investigate how administering multiple doses of tropisetron, a partial agonist of nAChR, for 1 day would affect cognitive deficits and P50 inhibition deficits in SCZ patients. We randomized 40 SCZ non-smokers into a double-blind clinical trial with four groups: placebo, $5 \mathrm{mg} / \mathrm{d}, 10 \mathrm{mg} / \mathrm{d}$, and $20 \mathrm{mg} / \mathrm{d}$ of oral tropisetron. Their P50 ratios were all more than 0.5 and they took risperidone at 3-6 mg/day for at least a month before participating in the experiment. We measured the Repeatable Battery for the Assessment of Neuropsychological Status (RBANS) and P50 inhibition before and one day after treatment. After one day of treatment, the total RBANS scores of the $20 \mathrm{mg}$ and $5 \mathrm{mg}$ tropisetron groups, and the immediate memory of the $10 \mathrm{mg}$ group were significantly higher than placebo group. The P50 ratio was smaller in the $5 \mathrm{mg}$ and $10 \mathrm{mg}$ groups than in the placebo group (both $p<0.05$ ) after treatment. Furthermore, the improvement in RBANS total score was correlated with increased S1 latency $(p<0.05)$, and the increase in immediate memory score was correlated with decreased S2 amplitude. One day of treatment with tropisetron improved both cognitive and P50 inhibition deficits, suggesting that longer term treatment with a7 nAChR agonists for these deficits in SCZ may be promising.

Neuropsychopharmacology (2020) 45:1362-1368; https://doi.org/10.1038/s41386-020-0685-0

\section{INTRODUCTION}

Impaired neurocognitive function is a core feature of schizophrenia (SCZ) [1] affecting memory [2, 3], executive function [4, 5], and attention [6]. Cognitive impairment of SCZ begins before the onset and persists for many years [7-9], leading to social dysfunction and a decline in overall quality of life [10, 11]. Cognitive impairment therefore has become a treatment target for SCZ research [12]. However, the pathophysiological mechanisms of cognitive deficits in SCZ patients remain unclear. Sensory gating is a mechanism that filters stimuli, suppresses irrelevant stimuli and protects the integrity of cognitive function [13, 14]. Sensory gating deficits appear to be sensitive biomarkers of cognitive impairment in SCZ patients [15-17]. Abnormalities in the P50 component of the electroencephalogram (EEG) recorded during repeated auditory stimuli can assess this inhibition deficit [17-19]. The neurochemical mechanism for this deficit appears to be through a genetic polymorphism in the $15 q 14$ locus of the gene coding for the a7 nicotinic acetylcholine receptor (nAChR), and agonists for this receptor are considered potential therapies to improve the sensory gating and cognitive deficits of SCZ patients [20-22].

Tropisetron, a commonly used medicine against nausea and vomiting caused by chemotherapy and surgical anesthesia, is both a potent serotonin-3 receptor antagonist and a high-affinity partial agonist of the nAChR [23-25]. Due to its nAChR activity, tropisetron has improved auditory inhibition and cognitive deficits in mice [26-28]. In patients with SCZ, $10 \mathrm{mg}$ per day of tropisetron significantly enhanced P50 deficiencies and cognitive deficits [23$25,29]$. In our previous study, we demonstrated that daily doses of tropisetron at 5,10 , and $20 \mathrm{mg}$ significantly improved overall cognitive deficits and P50 inhibition defects after 10 days of treatment [30].

The primary goal of this study was to compare the immediate effects of either 5, 10, or $20 \mathrm{mg}$ of tropisetron on cognitive and sensory gating deficits in patients with chronic SCZ after 1 day of treatment. Since a number of previous studies demonstrated the effectiveness of the $10 \mathrm{mg} /$ day dose $[23,30]$, we also considered 5 and $20 \mathrm{mg} /$ day to examine the dose-response effects. Since risperidone does not have significant $\mathrm{nAChR}$ agonism or $5-\mathrm{HT} 3$ receptor antagonism [31], we used it as an antipsychotic in this study. At the same time, we sought to examine the relationship between changes in sensory gating and cognitive improvement to verify the hypothesis that sensory gating acts on cognition by activating nAChRs.

\section{METHOD}

Participants

We recruited 40 non-smoking hospitalized patients with SCZ from the Shantou University Mental Health Center. Trained psychiatrists

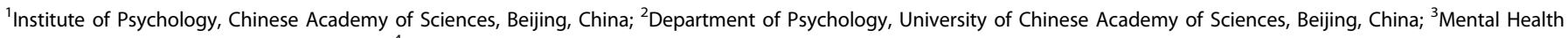

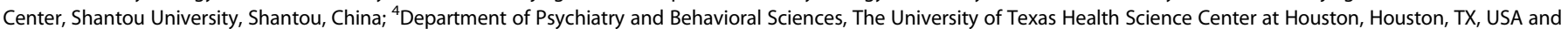
${ }^{5}$ Department of Psychiatry and Behavioral Sciences, Baylor College of Medicine, Houston, TX, USA Correspondence: Xiang Yang Zhang (zhangxy@psych.ac.cn)

Received: 15 March 2020 Revised: 17 April 2020 Accepted: 20 April 2020 Published online: 29 April 2020 
Table 1. Demographic data in four groups.

\begin{tabular}{lcccrrr}
\hline Characteristic and Measure & Placebo $(N=10)$ & $5 \mathrm{mg}(N=10)$ & $10 \mathrm{mg}(N=10)$ & $20 \mathrm{mg}(N=8)$ & $X^{2}$ or $F$ & $p$ \\
\hline Sex (M/F) & $7 / 3$ & $8 / 2$ & $7 / 3$ & $7 / 1$ & 1.070 & 0.784 \\
Age (years) & $36.50 \pm 10.61$ & $29.60 \pm 8.90$ & $26.30 \pm 5.25$ & $31.50 \pm 9.93$ & 1.188 & 0.283 \\
Education (years) & $7.40 \pm 2.07$ & $8.30 \pm 3.34$ & $8.00 \pm 1.89$ & $6.63 \pm 1.51$ & 0.786 & 0.381 \\
Duration of illness (months) & $73.71 \pm 77.30$ & $48.88 \pm 38.78$ & $50.25 \pm 47.21$ & $40.25 \pm 47.76$ & 1.417 & 0.242 \\
Risperidone dose at baseline (mg/day) & $4.64 \pm 0.86$ & $4.44 \pm 1.86$ & $4.72 \pm 1.24$ & $6.60 \pm 1.68$ & 9.039 & $<0.01$ \\
Risperidone dose at day 1 (mg/day) & $4.91 \pm 1.20$ & $5.66 \pm 1.47$ & $5.17 \pm 1.06$ & $7.41 \pm 1.79$ & 12.26 & $<0.01$ \\
\hline
\end{tabular}

used DSM-IV for structured clinical interviews for diagnosis (SCID) to make a diagnosis. All participants were Han Chinese, aged between 20 and 55 years old. Prior to participation in this study, participants continued to receive a steady dose of oral risperidone (3-6 mg/day) for more than 1 month without taking any other antipsychotic medications. All participants had sensory gating defects with P50 ratios $>0.5$. We excluded participants with cardiovascular or neurological disorders, a history of head trauma with a loss of consciousness, physical abnormalities, a DSM-IV dependent substance disorder, or a current mood or anxiety disorder. All participants had normal hearing acuity (subjective hearing threshold of $40 \mathrm{~dB}$ ).

All subjects went through a complete examination of their medical history, physical and laboratory indicators, and electrocardiogram. No one had alcohol or other substance dependence problems. A power analysis with the parameters of $0.85(P<0.05)$, and a medium effect size showed that our sample of 40 participants was adequate. All participants signed an informed consent form after fully understanding the procedure. The institutional review board of the Mental Health Center of Shantou University approved this study.

Clinical treatment

The experiment started with a 2-week placebo lead-in introduction. We performed sensory gating screening on 62 patients, yielding 40 patients with a P50 ratio $>0.5$. Patients with sensory gating defects compared with patients without sensory gating defects did not have any differences in demographic characteristics or disease duration (all $p>0.05$ ). The 40 participants were randomized into four groups of ten each, receiving either placebo, $5 \mathrm{mg}, 10 \mathrm{mg}$, or $20 \mathrm{mg}$ per day of tropisetron (Navoban, Novartis Pharmaceuticals, Ltd., Beijing). All capsules looked the same, and participants took them once a day. Participants also took a steady dose of 3-6 mg of risperidone per day during the experiment and did not take other antipsychotic drugs.

\section{P50 testing}

We recorded the P50 auditory evoked potentials at baseline and one hour after taking tropisetron or placebo. The electrophysiology test used a signal generator and a digital 40-channel EBNeuro Sirius EEG system (EBNeuro, Florence, Italy) data acquisition system. According to previous studies [32], participants sat in a chair and focused on a fixed point. A $90-\mathrm{dB}$ pulse stimulation signal lasting $0.1 \mathrm{~ms}$ generated an event-related potential waveform. A meta-analysis previously reported that the best sound intensity for stimulation was between 85 and $90 \mathrm{~dB}$ [33], consistent with the recommendation to use a standard stimulus of $50 \mathrm{~dB}$ above the hearing threshold. Each trial showed a pair of stimuli with an inter-stimulus interval of $500 \mathrm{~ms}$ and an inter-pair interval of $10 \mathrm{~s}[30,32,34,35]$. The number of trials was 32. Each epoch lasted $1000 \mathrm{~ms}$, including $100 \mathrm{~ms}$ before the first stimulation, $500 \mathrm{~ms}$ between stimuli, and $400 \mathrm{~ms}$ after the second stimulation. The event-related potential response was amplified and band-pass filtered by a $0.1-300 \mathrm{~Hz}$ analog filter and a $50 \mathrm{~Hz}$ notch filter. The sample rate was $512 \mathrm{~Hz}$. The data were analyzed offline, with $10-50 \mathrm{~Hz}, 12 \mathrm{~dB} /$ octave roll-off bandpass filtering. We excluded from the analysis any value greater than $\pm 50 \mu \mathrm{V}$ at any electrode. We recorded eye movements via two electrooculography (EOG) with $\mathrm{Ag} / \mathrm{AgCl}$ disc electrodes placed at the outer canthus and below the right eye. We corrected the ocular artifact using a regression method provided by EBNeuro digital EEG analysis and processing system. We referenced recordings to the left temporal apophysis and reduced the impedance to $<5 \mathrm{k} \Omega$. Throughout the process, we continued to provide $70 \mathrm{~dB}(\mathrm{~A})$ broadband white noise to avoid interference by background noise.

We calculated the P50 at the electrode sites of the vertex ( $C Z$ site) as the ratio of the S2 amplitude to the S1 amplitude [30, 32]. The S1 was the maximum amplitude within $30-90 \mathrm{~ms}$ after the first stimulus occurred. The S2 was the maximum amplitude closest to the S1 latency after the second stimulus.

Two independent neurophysiologists analyzed the P50 test results, unaware of the participants' status. If the results they reported were inconsistent, the two examiners reanalyzed the results together to reach a consensus.

\section{Cognitive performance}

We used the Repeatable Battery for the Assessment of Neuropsychological Status (RBANS) [36] to assess cognitive function. The RBANS includes 12 sub-tests for calculating a total score and five age-adjusted index scores, including immediate memory, visuospatial/constructional, language, attention, and delayed memory. Our team translated this tool into Chinese and established its clinical validity and test-retest reliability in healthy subjects and SCZ patients $[37,38]$. We performed the RBANS and P50 tests on the same day.

\section{Statistical analysis}

We performed repeated measures (RM) multivariate analysis of variance (MANOVA) on RBANS total score and subscale scores, including a between-group medicine factor (placebo, 5, 10, 20 mg) and a within-group time factor (baseline and 1-day). The normal distribution was evaluated by the Kolmogorov Smirnov specimen test $(p>0.05)$. Levene's test was used to assess variance homogeneity $(p>0.05)$, and Mauchly's test for the assumption of sphericity $(p>0.05)$. We used age, education, gender, duration of disease, and risperidone dose as covariates if the interactive effect of medicine $X$ time in RM MANOVA was significant. When the overall treatment effect was significant, we used the covariance analysis (ANCOVA) with the baseline score as the covariate, and then performed the Dunnett test to compare the differences between the dose groups. If the overall treatment effect was significant, we compared the RBANS total and subscale scores for each dose group with the placebo group by covariance analysis (ANCOVA). We used a similar RM ANOVA to analyze the P50 data. We performed Pearson correlations between the changes in RBANS and P50 gating ratios. Other statistical analyses included analysis of variance (ANOVA) for continuous variables, and chi-square test for categorical variables. To correct for multiple tests, we used the Bonferroni test, because it allows for tight control of multiple tests and reduces the probability of false positives type I errors. Since we performed six comparisons 
in the four groups, the adjusted level of significance with the Bonferroni test was set to $p=0.05 / 6=0.0083$. We used $\mathrm{R}$ version 3.5.2 (http://cran.r-project.org) [39] to do all the statistical analyses with a two-tailed significance level of $\leq 0.05$ and expressed data as mean \pm standard deviation.

\section{RESULTS}

Demographic characteristics and duration of illness showed no significant differences between the four treatment groups (see Table 1). Risperidone doses differed between groups at baseline $(p<0.01)$. Therefore, subsequent analysis included risperidone dose as a covariate.

Effects of tropisetron treatment on cognitive performance The RBANS total and index scores (Table 2) were not different between the treatment groups at baseline (all $p>0.05$ ). The RBANS total score $(F=3.19, \mathrm{df}=3,34, p<0.05)$ and the immediate memory score $(F=3.55, \mathrm{df}=3,34, p<0.05)$ showed significant tropisetron dose-by-time effects. When the effects were examined by adding age, education, sex, duration of disease, and risperidone dose as covariates to the RM MANOVA, the immediate memory $(F=3.52, \mathrm{df}=3,29, p<0.05)$ was still significant, while the total score $(F=2.33$, df $=3,29, P<0.1)$ was not.

Using the baseline value as covariate, the ANCOVA analysis showed a main effect of doses on the total RBANS score $(F=3.81$, $\mathrm{df}=3,29, p<0.05)$. Dunnett post hoc testing showed that the total RBANS score of the $20 \mathrm{mg}(p=0.02$, Cohen's $d=1.36)$ and 5 $\mathrm{mg}(p=0.018$, Cohen's $d=1.30)$ tropisetron groups was significantly higher than that of the placebo group after one day of treatment. Further, dose had a main effect on immediate memory $(F=3.92, \mathrm{df}=3,29, p<0.05)$, showing that the immediate memory score was significantly higher for the $10 \mathrm{mg}$ than the placebo group $(p=0.008$, Cohen's $d=1.44)$, which remained significant after a Bonferroni correction. However, the three tropisetron dose groups did not differ in the RBANS total score and index scores (all $p>0.05$ ).

Effects of tropisetron treatment on P50 Inhibition

The four groups showed no significant differences in the P50 components at baseline (all $p>0.05$ ), and the P50 components showed no significant effect of the dose-by-time $(p>0.05)$ (see Table 3).

Using the baseline P50 ratio as a covariate, we showed a main effect of tropisetron doses on the $\mathrm{P} 50$ ratio $(F=3.39$, df $=3,29$, $p<0.05)$. Dunnett post hoc testing showed that the P50 ratio was smaller in the $5 \mathrm{mg}$ group ( $p=0.02$, Cohen's $d=-1.28)$ and the $10 \mathrm{mg}$ group $(p=0.035$, Cohen's $d=-1.17)$ than that in the placebo group. However, none of these differences passed the Bonferroni correction (Bonferroni corrected $p=0.0083$ ), and the three tropisetron dose groups were not different in P50 components (all $p>0.05$ ).

Relationship between changes in P50 inhibition and cognitive performance

After treatment, the increase in the latency of S1 (Day one score minus baseline) was associated with the improvement in RBANS total score $(r=0.32, p<0.05$, Fig. 1$)$, but the decrease in S2 amplitude was not, although S2 amplitude change had a trend toward significant correlation with the increase in immediate memory score $(r=0.32, p$ $=0.053$ ). Additionally, the 10 and $20 \mathrm{mg}$ doses of tropisetron showed no difference in effects on the P50 gating ratio, which is characteristic of partial agonists at high doses (Fig. 2).

\section{DISCUSSION}

This study found that one dose of 5,10 , and $20 \mathrm{mg}$ tropisetron treatment significantly improved immediate memory. The

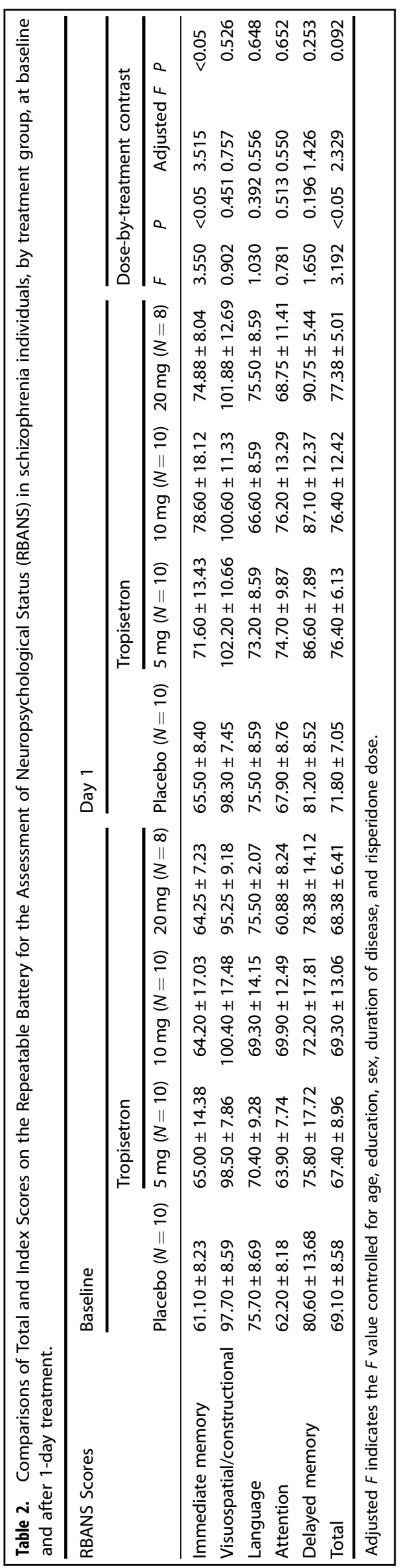




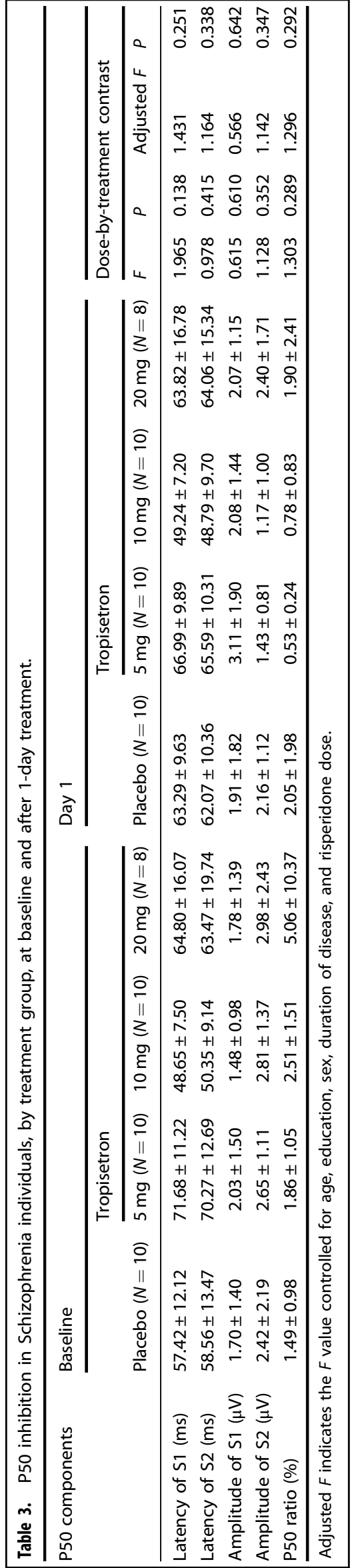

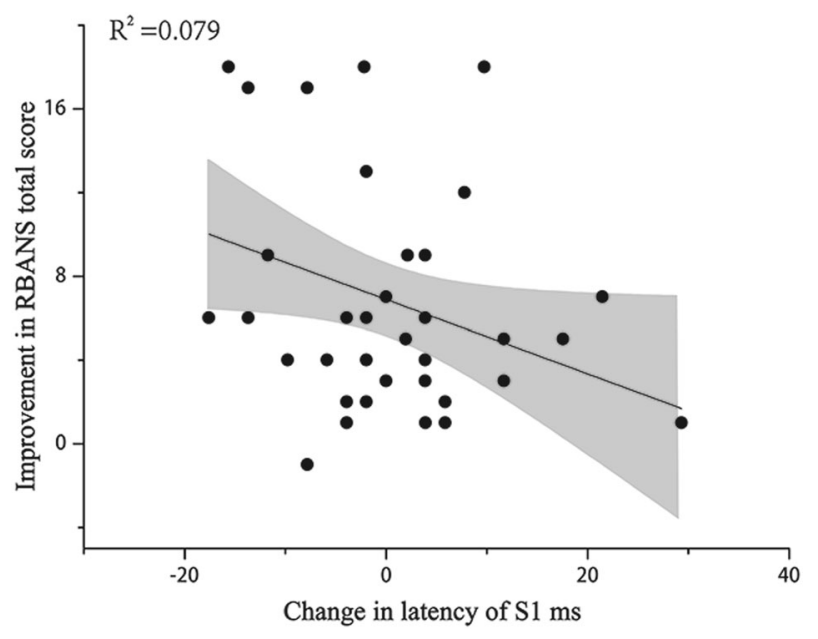

Fig. 1 Association between the improvement in the total score of RBANS and the change in the latency of S1 after treatment. After treatment, the higher improvement in RBANS total score measured by Repeatable Battery for the Assessment of Neuropsychological Status (RBANS) was associated with a larger increase in S1 latency (day one score minus baseline).

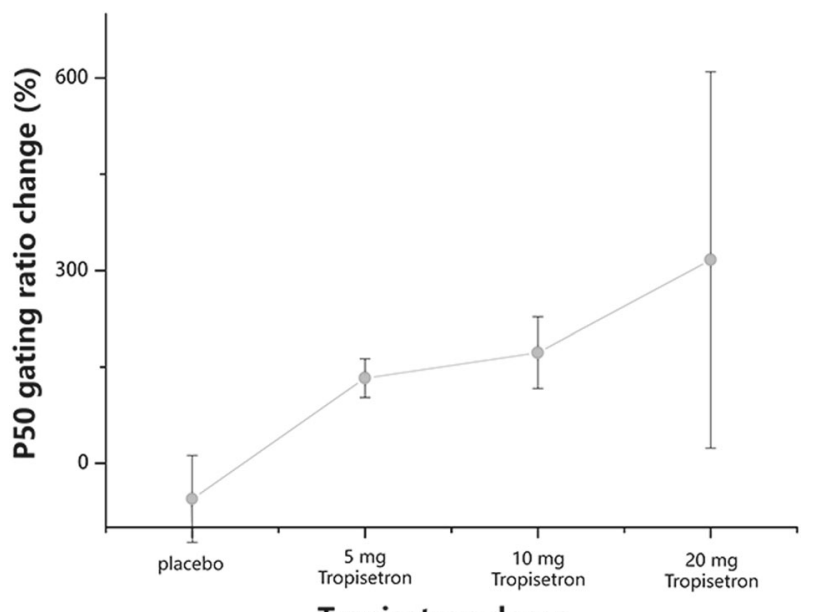

Tropisetron doses

Fig. 2 Changes in P50 Gating Ratio in Patients With Schizophrenia After 1-Day Treatment With Tropisetron or Placeboa. The changes in P50 gating ratio after 1-day treatment with placebo or tropisetron show flattened dose-response curves.

improvement in immediate memory in the $10 \mathrm{mg}$ group was more significant and passed the Bonferroni correction. A previous study found that after ten days of treatment, taking $10 \mathrm{mg}$ of tropisetron per day was associated with increased immediate memory, while taking $20 \mathrm{mg}$ per day was associated with increased delayed memory [30]. Another study showed that after 2 weeks of treatment, $10 \mathrm{mg}$ of tropisetron per day improved sustained visual attention [23]. Consistent with previous studies, we found that only one-dose of tropisetron treatment improved cognitive performance, especially immediate memory in patients with chronic SCZ.

The study also found improved P50 inhibition deficits in the active treatment group, showing a reduction in both the $\mathrm{P} 50$ ratio and the S2 amplitude. However, the three-tropisetron dose groups did not differ in improving P50 inhibition. This is consistent with previous studies, which reported that tropisetron improved P50 deficiency in patients with SCZ within a limited dose range [23$25,30]$. In addition, our current study also demonstrated that only 
1366

one-day of treatment with tropisetron may improve neurological function.

Interestingly, we found that there was a significant association between cognitive improvement and improvement in P50 inhibition defects in SCZ patients. Specifically, the increase in the total RBANS score was associated with the change in S1 latency. Our findings are consistent with two previous studies, which found that the increase in the total of RBANS score was associated with a change in the $\mathrm{P} 50$ ratio in SCZ patients treated by low-dose DMXB-A (a nicotinic agonist) or by 10 days of tropisetron [30,40]. However, another small sample-size study did not find a correlation between cognitive improvement and P50 composition in SCZ patients treated with tropisetron [23].

Tropisetron appears to improve P50 inhibition and cognitive deficits in patients with SCZ through its excitatory effects on a7 nAChRs [23, 30, 41, 42]. Mouse studies found that tropisetron improved inhibition processing defects [26-28]. Similarly, other studies found that the $\mathrm{nAChR}$ agonist DMXB-A improved P50 inhibition defects and cognitive deficits in patients with SCZ [2325 , 43]. However, tropisetron may improve cognitive performance due to its potent antagonism of the 5-HT3 receptor. However, in a mouse model of phencyclidine-induced $\mathrm{SCZ}$, tropisetron and the nAChR agonist SSR180711 improved cognitive deficits, whereas the 5-HT3 receptor antagonist ondansetron did not [44, 45]. Moreover, when taking the a7 nAChRs antagonist methyllycaconitine, the effects of the agonists tropisetron and SSR180711 were blocked $[44,45]$. A previous PET study also demonstrated that oral administration of tropisetron at 5, 10, or $20 \mathrm{mg}$ acted on a7 $\mathrm{nAChRs}$ in a dose-dependent manner [46].

More than $80 \%$ of patients with chronic SCZ depend on nicotine, which is two to four times that of the general population or other patients with severe mental disorders [45-49]. The problem of nicotine abuse usually exists before the onset of SCZ [50]. Studies have shown that the appropriate amount of nicotine can improve the cognitive process of healthy people, as well as the attention and memory of SCZ [51-55]. The abnormal P50 ratio in SCZ is related to the increased incidence of ChRNA7 allele variation in the $a 7$ nicotine acetylcholine receptor (NAChR) subunit gene [56]. The increase in smoking rate in patients with SCZ is related to ChRNA7 [57]. Smoking can temporarily improve the P50 inhibition deficits in SCZ patients [58]. Nicotine can also normalize cholinergic nerve conduction defects in patients with SCZ [58]. Smoking in SCZ patients has also been explained as an attempt to self-treat underlying pathophysiological gating mechanisms [59-61]. In this study, smokers were not required to quit from nicotine before the test. Therefore, the results were statistically significant and robust.

Risperidone is a commonly used drug in SCZ patients and contains benzoxazole and piperidine, which are antagonists of the D1 (D1, D5) and D2 (D2, D3, D4) receptor families, acting on the central nervous system, especially on the mesolimbic, prefrontal corticolimbic, and tuberoinfundibular pathways [62]. Also, risperidone affects the alpha 2 adrenergic receptors, which affect positive symptoms, negative symptoms, mood and cognition [63]. Although most second-generation antipsychotic drugs may have cognitive improvement effects [64], risperidone has been shown to be ineffective against P50 inhibition defects in patients with chronic SCZ [65-68]. Before participating in this study, participants were required to continue to receive a stable dose of oral risperidone (3-6 mg/day) for more than a month without taking any other antipsychotic drugs. The study also set up a placebo group. Therefore, the drug has little effect on the results of this study. However, we still need to explore the effects of risperidone on cognition and P50 ratio in first-episode, drug-naive SCZ patients.

Alzheimer's disease (AD) is the most common age-related neurodegenerative disease [69]. There is accumulating evidence that the interaction between amyloid- $\beta$ peptides $(A \beta)$ and $\alpha 7$
nAChRs is indispensable in the pathological process of $\operatorname{AD}[27,70]$. The interaction between $A \beta$ peptides and $\alpha 7 n A C h R s$ may cause $A \beta$ deposition and inflammation in the brain, leading to the loss of cholinergic neurons and a7 nAChRs [69]. These neurotoxic events may promote cognitive decline, which in turn leads to mild cognitive impairment $(\mathrm{MCl})$ and ultimately $A D$ [69]. Tropisetron binds to a7 nAChRs as an agonist, to serotonin 5-HT3 receptor as an antagonist, and to APP with sub-micromolar affinity [27]. The interaction of tropisetron with $5-\mathrm{HT} 3$ receptors also plays a role in the anti-inflammatory and neuroprotective effects against $A \beta$ induced neurotoxicity [70]. Taken together, tropisetron may be a potential drug for the treatment of $A D$ if it is used during $\mathrm{MCl}$ or early stages of AD. It may reduce cognitive impairment and P50 inhibition defects in $A D$ patients, but the specific effect still needs to be further studied.

While these rapid results are exciting, this study has some limitations. First, the sample size was relatively small, and the findings in our current study should be confirmed in larger sample size of studies. Second, the P50 component represents only the neural activity that occurs at the surface of the brain. Cognitive deficits in schizophrenia may involve activity in deeper brain regions. Third, cholinergic regulation may affect cognitive performance and P50 testing. However, we did not collect data on smoking status in this study, which should be remedied in future studies. Therefore, future research should use a larger sample size, including data on smoking status and other techniques, such as functional magnetic resonance imaging.

In summary, our results indicate that one dose of tropisetron treatment can improve cognitive deficits and P50 inhibition deficits in patients with SCZ. Interestingly, improvements of these two deficits showed a significant correlation, likely due to tropisetron's effect on a7 nAChRs. Future studies can extend these findings using a large sample of patients with first-episode of SCZ, including a variety of data such as smoking status, and a combination of techniques such as ERP and functional MRI to investigate the effects of tropisetron on cognitive and sensory gating deficits.

\section{FUNDING AND DISCLOSURE}

Funding for this study was provided by the National Natural Science Foundation of China (81371477) and the CAS Pioneer Hundred Talents Program. No conflict of interest was disclosed for each author.

\section{AUTHOR CONTRIBUTIONS}

$\mathrm{LX}$ and $\mathrm{XYZ}$ performed analysis, made interpretation of data and drafted the manuscript. $\mathrm{LL}$ and $\mathrm{XH}$ made contributions to the acquisition of clinical and P50 data for the work. DW, GW, JW, HZ, HX, YT, QD, and LW helped draft the work and revise it critically. HEW, CC, and TRK helped to interpret the data and revised the paper significantly. All authors made significant contributions to the paper to assess the important intellectual content, read and approved the paper.

\section{REFERENCES}

1. Kanchanatawan B, Hemrungrojn S, Thika S, Sirivichayakul S, Ruxrungtham K, Carvalho AF, et al. Changes in Tryptophan Catabolite (TRYCAT) pathway patterning are associated with mild impairments in declarative memory in schizophrenia and deficits in semantic and episodic memory coupled with increased false-memory creation in deficit schizophrenia. Mol Neurobiol. 2018;55:5184-201.

2. Vohringer PA, Barroilhet SA, Amerio A, Reale $M L$, Alvear $K$, Vergne $D$, et al. Cognitive impairment in bipolar disorder and schizophrenia: a systematic review. Front Psychiatry. 2013;4:87.

3. Khalil AH, El-Meguid MA, Bastawy M, Rabei S, Ali R, Abd Elmoneam MHE. Correlating cognitive functions to symptom domains and insight in Egyptian patients with schizophrenia. Int J Soc Psychiatry. 2020;66:240-248.

4. Simonsen $C$, Sundet $K$, Vaskinn A, Birkenaes AB, Engh JA, Faerden A, et al. Neurocognitive dysfunction in bipolar and schizophrenia spectrum disorders depends on history of psychosis rather than diagnostic group. Schizophr Bull. 2011;37:73-83. 
5. Giraldo-Chica M, Rogers BP, Damon SM, Landman BA, Woodward ND. Prefrontalthalamic anatomical connectivity and executive cognitive function in schizophrenia. Biol Psychiatry. 2018;83:509-17.

6. Hoonakker M, Doignon-Camus N, Bonnefond A. Sustaining attention to simple visual tasks: a central deficit in schizophrenia? A systematic review. Ann N. Y Acad Sci. 2017;1408:32-45.

7. Paquin K, Wilson AL, Cellard C, Lecomte T, Potvin S. A systematic review on improving cognition in schizophrenia: which is the more commonly used type of training, practice or strategy learning? BMC Psychiatry. 2014;14:139.

8. Schreiber R, Newman-Tancredi A. Improving cognition in schizophrenia with antipsychotics that elicit neurogenesis through 5-HT(1A) receptor activation. Neurobiol Learn Mem. 2014;110:72-80.

9. Neu P, Gooren T, Niebuhr U, Schlattmann P. Cognitive impairment in schizophrenia and depression: a comparison of stability and course. Appl Neuropsychol Adult. 2019;26:215-28.

10. Peralta V, Cuesta MJ. Social cognition in schizophrenia: the relevance of early detection and intervention. Sist Sanit Navar. 2017:40:173-75.

11. Dziwota E, Stepulak MZ, Wloszczak-Szubzda A, Olajossy M. Social functioning and the quality of life of patients diagnosed with schizophrenia. Ann Agric Environ Med. 2018;25:50-5.

12. Davidson M. Cognitive impairment as a diagnostic criterion and treatment target in schizophrenia. World Psychiatry. 2019;18:171.

13. Hamling KR, Schoppik D. Sensory gating: cellular substrates of surprise. Curr Biol. 2018;28:R871-3.

14. Lei Y, Ozdemir RA, Perez MA. Gating of sensory input at subcortical and cortical levels during grasping in humans. J Neurosci. 2018;38:7237-47.

15. Toyomaki A, Hashimoto N, Kako Y, Tomimatsu Y, Koyama T, Kusumi I. Different P50 sensory gating measures reflect different cognitive dysfunctions in schizophrenia. Schizophr Res Cogn. 2015;2:166-9.

16. Xia L, Yuan L, Du XD, Wang D, Wang J, Xu H, et al. $P 50$ inhibition deficit in patients with chronic schizophrenia: relationship with cognitive impairment of MATRICS consensus cognitive battery. Schizophr Res. 2020;215:105-12.

17. Schubring D, Popov T, Miller GA, Rockstroh B. Consistency of abnormal sensory gating in first-admission and chronic schizophrenia across quantification methods. Psychophysiology. 2018;55:1-13.

18. Chien YL, Hsieh MH, Gau SS. P50-N100-P200 sensory gating deficits in adolescents and young adults with autism spectrum disorders. Prog Neuropsychopharmacol Biol Psychiatry. 2019;95:109683.

19. Karkal R, Goyal N, Tikka SK, Khanande RV, Kakunje A, Khess CR. Sensory gating deficits and their clinical correlates in drug-free/drug-naive patients with schizophrenia. Indian J Psychol Med. 2018;40:247-56.

20. Koukouli F, Rooy M, Tziotis D, Sailor KA, O'Neill HC, Levenga J, et al. Nicotine reverses hypofrontality in animal models of addiction and schizophrenia. Nat Med. 2017;23:347-54.

21. Jones C. alpha7 nicotinic acetylcholine receptor: a potential target in treating cognitive decline in schizophrenia. J Clin Psychopharmacol. 2018;38:247-49.

22. Wadenberg MG, Manetti D, Romanelli MN, Arias HR. Significance of the nicotinic alpha7 receptor in cognition and antipsychotic-like behavior in the rat. Behav Brain Res. 2017;333:129-34.

23. Shiina A, Shirayama $Y$, Niitsu T, Hashimoto T, Yoshida T, Hasegawa T, et al. A randomised, double-blind, placebo-controlled trial of tropisetron in patients with schizophrenia. Ann Gen Psychiatry. 2010;9:27.

24. Hashimoto $\mathrm{K}$. Targeting of $\mathrm{a} 7$ nicotinic acetylcholine receptors in the treatment of schizophrenia and the use of auditory sensory gating as a translational biomarker. Curr Pharm Des. 2015;21:3797-806.

25. Noroozian M, Ghasemi S, Hosseini SM, Modabbernia A, Khodaie-Ardakani MR, Mirshafiee $\mathrm{O}$, et al. A placebo-controlled study of tropisetron added to risperidone for the treatment of negative symptoms in chronic and stable schizophrenia. Psychopharmacology (Berl). 2013;228:595-602.

26. Poddar I, Callahan PM, Hernandez CM, Yang X, Bartlett MG, Terry AV Jr. Tropisetron enhances recognition memory in rats chronically treated with risperidone or quetiapine. Biochem Pharm. 2018;151:180-7.

27. Spilman P, Descamps O, Gorostiza O, Peters-Libeu C, Poksay KS, Matalis A, et al. The multi-functional drug tropisetron binds APP and normalizes cognition in a murine Alzheimer's model. Brain Res. 2014;1551:25-44.

28. Hashimoto K, lyo M, Freedman R, Stevens KE. Tropisetron improves deficient inhibitory auditory processing in DBA/2 mice: role of alpha 7 nicotinic acetylcholine receptors. Psychopharmacol (Berl). 2005;183:13-9.

29. Koike K, Hashimoto K, Takai N, Shimizu E, Komatsu N, Watanabe H, et al. Tropisetron improves deficits in auditory P50 suppression in schizophrenia. Schizophr Res. 2005:76:67-72.

30. Zhang $X Y$, Liu L, Liu S, Hong $X$, Chen DC, Xiu MH, et al. Short-term tropisetron treatment and cognitive and P50 auditory gating deficits in schizophrenia. Am J Psychiatry. 2012;169:974-81.
31. Chopko TC, Lindsley CW. Classics in chemical neuroscience: risperidone. ACS Chem Neurosci. 2018;9:1520-29.

32. Hong X, Chan RC, Zhuang X, Jiang T, Wan X, Wang J, et al. Neuroleptic effects on P50 sensory gating in patients with first-episode never-medicated schizophrenia. Schizophr Res. 2009;108:151-7.

33. de Wilde OM, Bour L, Dingemans P, Koelman J, Linszen D. A meta-analysis of P50 studies in patients with schizophrenia and relatives: differences in methodology between research groups. Schizophr Res. 2007;97:137-51.

34. Dolu N, Suer C, Ozesmi C. A comparison of the different interpair intervals in the conditioning-testing P50 paradigms. Int J Psychophysiol. 2001;41:265-70.

35. Sánchezmorla EM, Santos JL, Aparicio A, Garcíajiménez MÁ, Soria C, Arango C. Neuropsychological correlates of P50 sensory gating in patients with schizophrenia. Schizophr Res. 2013;143:102-6.

36. Randolph C, Tierney MC, Mohr E, Chase TN. The Repeatable Battery for the Assessment of Neuropsychological Status (RBANS): preliminary clinical validity. J Clin Exp Neuropsychol. 1998;20:310-9.

37. Zhang BH, Tan YL, Zhang WF, Wang ZR, Yang GG, Shi C, et al. Repeatable battery for the assessment of neuropsychological status as a screening test in Chinese: reliability and validity. Chin Ment Health J. 2008;22:865-69.

38. Cheng Y, Wu W, Wang J, Feng W, Wu X, Li C. Reliability and validity of the Repeatable Battery for the Assessment of Neuropsychological Status in community-dwelling elderly. Arch Med Sci. 2011;7:850-7.

39. Team RDC. R: A Language and Environment for Statistical Computing. Vienna: R Foundation for Statistical Computing. 2015.

40. Freedman R, Olincy A, Buchanan RW, Harris JG, Gold JM, Johnson L, et al. Initial phase 2 trial of a nicotinic agonist in schizophrenia. Am J Psychiatry. 2008;165:1040-7.

41. Ishikawa M, Hashimoto K. a7 nicotinic acetylcholine receptor as a potential therapeutic target for schizophrenia. Curr Pharm Des. 2011;17:121-29.

42. Boggs DL, Carlson J, Cortes-Briones J, Krystal JH, D'Souza DC. Going up in smoke? A review of $n A C h R s$-based treatment strategies for improving cognition in schizophrenia. Curr Pharm Des. 2014;20:5077-92.

43. Hamilton HK, D'Souza DC, Ford JM, Roach BJ, Kort NS, Ahn KH, et al. Interactive effects of an $\mathrm{N}$-methyl-d-aspartate receptor antagonist and a nicotinic acetylcholine receptor agonist on mismatch negativity: implications for schizophrenia. Schizophr Res. 2018;191:87-94.

44. Hashimoto $K$, Ishima $T$, Fujita $Y$, Matsuo $M$, Kobashi $T$, Takahagi $M$, et al. Phencyclidine-induced cognitive deficits in mice are improved by subsequent subchronic administration of tropisetron: role of a 7 nicotinic receptors. Biol Psychiatry. 2008;553:191-5.

45. Hashimoto K, Ishima T, Fujita Y, Matsuo M, Kobashi T, Takahagi M, et al. Phencyclidine-induced cognitive deficits in mice are improved by subsequent subchronic administration of the novel selective alpha7 nicotinic receptor agonist SSR180711. Biol Psychiatry. 2008;63:92-7.

46. Ishikawa M, Sakata M, Toyohara J, Oda K, Ishii K, Wu J, et al. Occupancy of a7 nicotinic acetylcholine receptors in the brain by tropisetron: a positron emission tomography study using $[(11) \mathrm{C}] \mathrm{CHIBA}-1001$ in healthy human subjects. Clin Psychopharmacol Neurosci. 2011;9:111-6.

47. Sagud M, Vuksan-Cusa B, Jaksic N, Mihaljevic-Peles A, Rojnic Kuzman M, Pivac N Smoking in Schizophrenia: an Updated Review. Psychiatr Danub. 2018;30(Suppl 4):216-23.

48. Fond G, Berna F, Andrianarisoa M, Godin O, Leboyer M, Brunel L, et al. Chronic low-grade peripheral inflammation is associated with severe nicotine dependence in schizophrenia: results from the national multicentric FACE-SZ cohort. Eur Arch Psychiatry Clin Neurosci. 2017;267:465-72.

49. Dickerson F, Origoni A, Schroeder J, Adamos M, Katsafanas E, Khushalani S, et al. Natural cause mortality in persons with serious mental illness. Acta Psychiatr Scand. 2018;137:371-79.

50. Dickerson F, Stallings CR, Origoni AE, Vaughan C, Khushalani S, Schroeder J, et al. Cigarette smoking among persons with schizophrenia or bipolar disorder in routine clinical settings, 1999-2011. Psychiatr Serv. 2013;64 44-50.

51. Zhang XY, Rao WW, Yu Q, Yu Y, Kou C, Tan YL, et al. Association of the manganese superoxide dismutase gene Ala-9Val polymorphism with age of smoking initiation in male schizophrenia smokers. Am J Med Genet Part B Neuropsychiatr Genet. 2016;171:243-49.

52. Zhang $X Y$, Li CB, Li M, Zheng YL, Zhang CX, Yan QZ, et al. Smoking initiation and schizophrenia: a replication study in a Chinese Han population. Schizophr Res. 2010;119:110-4.

53. Heishman SJ, Kleykamp BA, Singleton EG. Meta-analysis of the acute effects of nicotine and smoking on human performance. Psychopharmacol (Berl). 2010;210:453-69.

54. Campos MW, Serebrisky D, Castaldelli-Maia JM. Smoking and cognition. Curr Drug Abus Rev. 2016;9:76-9. 
55. Hong L, Schroeder M, Ross T, Buchholz B, Salmeron B, Wonodi I, et al. Nicotine enhances but does not normalize visual sustained attention and the associated brain network in schizophrenia. Schizophr Bull. 2011;37:416-25.

56. Zhang XY, Tan YL, Chen DC, Tan SP, Yang FD, Zunta-Soares GB, et al. Effects of cigarette smoking and alcohol use on neurocognition and BDNF levels in a Chinese population. Psychopharmacol (Berl). 2015;233:435-45.

57. Tan SP, Jie-Feng C, Fan FM, Zhao YL, Chen N, Fan HZ, et al. Smoking, MATRICS consensus cognitive battery and P50 sensory gating in a Han Chinese population. Drug Alcohol Depend. 2014;143:51-7.

58. Choueiry J, Blais CM, Shah D, Smith D, Fisher D, Illivitsky V, et al. Combining CDPcholine and galantamine: Effects of a selective alpha7 nicotinic acetylcholine receptor agonist strategy on P50 sensory gating of speech sounds in healthy volunteers. J Psychopharmacol. 2019;33:688-99.

59. Leon JD, Diaz FJ. A meta-analysis of worldwide studies demonstrates an association between schizophrenia and tobacco smoking behaviors. Schizophr Res. 2005;76:135-57.

60. Turan T, Dolu N, Ozsoy S, Kılıç C, BeșiRli A, Esel E. Effects of smoking on P50 waveform in schizophrenic patients. Klin Psikofarmakol Bul. 2009;19:227-35.

61. Winterer G. Why do patients with schizophrenia smoke? Curr Opin Psychiatry. 2010;23:112.

62. Liu H, Luo Q, Du W, Li X, Zhang Z, Yu R, et al. Cigarette smoking and schizophrenia independently and reversibly altered intrinsic brain activity. Brain Imaging Behav. 2018;12:1457-65.
63. Pesko MF, Baum CF. The self-medication hypothesis: evidence from terrorism and cigarette accessibility. Econ Hum Biol. 2016;22:94-102.

64. Kodaka F, Ito H, Takano H, Takahashi H, Arakawa R, Miyoshi M, et al. Effect of risperidone on high-affinity state of dopamine D2 receptors: a PET study with agonist ligand [11C](R)-2-CH3O-N-n-propylnorapomorphine. Int J Neuropsychopharmacol. 2011;14:83-9.

65. Hecht EM, Landy DC. Alpha-2 receptor antagonist add-on therapy in the treatment of schizophrenia; a meta-analysis. Schizophr Res. 2012;134:202-6.

66. Goldberg TE, Goldman RS, Burdick KE, Malhotra AK, Todd L, Patel RC, et al. Cognitive improvement after treatment with second-generation antipsychotic medications in first-episode schizophrenia: is it a practice effect? Arch Gen Psychiatry. 2007;64:1115.

67. Liu DT, Zhuo KM, Song ZH, Yan WU, Chen XS, Wang JJ, et al. Effect of risperidone on sensory gating P50 deficit in patients with schizophrenia. J Shanghai Jiaotong Univ. 2010;30:1525-29.

68. Xu L, Song L, Zhang M, Chen X, Wu R, Tang J. A study on the effect of risperidone on PPI and P50 Deficit in FirstEpisode and chronic patients with schizophrenia. J Int Psychl. 2016;4:577-80.

69. Hashimoto K. Tropisetron and its targets in Alzheimer's disease. Expert Opin Ther Targets. 2015;19:1-5

70. Rahimian R, Fakhfouri G, Ejtemaei Mehr S, Ghia JE, Genazzani AA, Payandemehr B, et al. Tropisetron attenuates amyloid-beta-induced inflammatory and apoptotic responses in rats. Eur J Clin Invest. 2013;43:1039-51. 